\title{
Undiagnosed acute HIV infection identified through RNA testing of pooled serum samples obtained during a dengue outbreak in São Paulo, Brazil
}

\author{
Elaine Monteiro Matsuda ${ }^{[1]}$, Daniela Rodrigues Colpas ${ }^{[2]}$, Norberto Camilo Campos $^{[3]}$, \\ Luana Portes Ozorio Coelho ${ }^{[3]}$, Andreia Moreira dos Santos Carmo ${ }^{[2]}$ \\ and Luís Fernando de Macedo Brígido ${ }^{[3]}$
}

[1]. Programa de Controle das DST/AIDS, Prefeitura Municipal de Santo André, Santo André, São Paulo, Brasil. [2]. Laboratório Regional, Instituto Adolfo Lutz, Governo do Estado de São Paulo, Santo André, São Paulo, Brasil. [3]. Centro de Virologia, Instituto Adolfo Lutz, São Paulo, São Paulo, Brasil.

\begin{abstract}
Introduction: Improving HIV diagnostics and treatment is necessary to end the AIDS epidemic. Pooled plasma can be used to identify patients with acute HIV disease, even before serological tests. During dengue outbreaks, patients having symptoms common to other acute viral diseases might seek medical care. Methods: We evaluated HIV RNA in pooled seronegative dengue samples. Results: After excluding individuals with a known HIV diagnosis, an HIV-1 prevalence of $0.73 \%$ [ $95 \%$ confidence interval (CI) $0.23-1.76 ; 4 / 546$ samples] was found. Conclusions: Promoting strategies to diagnose these individuals and provide them with medical treatment might be instrumental for controlling the HIV epidemic.
\end{abstract}

Keywords: HIV. Acute disease. Dengue.

Patients with incident human immunodeficiency virus (HIV) infection can present with symptoms that are common to other acute viral infections; these cases are often misdiagnosed ${ }^{1-3}$. Moreover, many patients do not seek care due to a variety of reasons, including the chronic limitations in accessibility to care in limited resource settings. During arbovirus outbreaks, a combination of increased awareness for sentinel symptoms and the temporary increase in healthcare capability to cope with the surge in disease cases might provide an opportunity to improve HIV diagnostics, in particular in patients who are in the acute phase of disease. Importantly, this phase in HIV disease is associated with increased transmissibility ${ }^{1}$. Moreover, increasing evidence indicates that early treatment benefit patients and the community ${ }^{4}$. Universal treatment is a strategic priority ${ }^{5}$ in the fight against the AIDS epidemic. However, diagnostic limitations regarding the acute phase of infection might hamper these efforts.

Pooled plasma has been shown to allow for the identification of patients in the acute phase of disease ${ }^{6-8}$. Since individual HIV RNA testing is expensive, a preliminary test of a mixture of plasma (pool) from different individuals could be performed. Subsequently, a plasma pool that tested positive can be tested to

Corresponding author: Dr. Luís Fernando de Macedo Brígido.

e-mail: lubrigido@gmail.com

Received 15 September 2016

Accepted 24 November 2016 identify the single sample testing positive for RNA or another marker of HIV infection.

We analyzed pooled samples of seronegative dengue cases that were collected during the 2015 outbreak in Santo André City, São Paulo metropolitan area, for HIV RNA to evaluate the feasibility of identifying HIV infection in individuals seeking medical care due to suspected arbovirus infection. In 2015, a total of 5,357 suspected dengue cases were reported to the Santo André Epidemiological Surveillance Department. The Santo André Regional Adolfo Lutz Laboratory received 3,119 samples to perform the dengue diagnostic tests. Of these, $1,976(63.4 \%)$ showed reactivity to the NS1 antigen or immunoglobulin $\mathrm{M}(\operatorname{IgM})$ in the enzyme-linked immunosorbent assay (ELISA). Of the 1,143 non-reactive samples, 570 were available for further testing. We used an electronic official data registry to identify cases of individuals who received antiretroviral treatment and/or had performed HIV monitoring laboratory tests (CD4 T cell counts or viral load determinations). Samples of 10 patients were excluded due to a previous HIV diagnosis. Of the 560 dengue-negative samples, 28 plasma pools were prepared with $100 \mu \mathrm{L}$ of plasma of 20 samples each (total $2 \mathrm{~mL}$ ) and maintained at $-70^{\circ} \mathrm{C}$ until HIV RNA was evaluated with Real Time HIV (Abbott, USA). Samples included in pools with detectable HIV RNA were analyzed separately for HIV RNA. Those with detectable viremia were also tested for serology (Imunoblot DPP HIV 1/2-biomanguinhos, Brazil) and sequenced (partial pol nested RT-PCR, followed 
by Big Dye, Life, USA). Sequences were evaluated for HIV genotyping(https://www.ncbi.nlm.nih.gov/projects/genotyping) and antiretroviral resistance (https://hivdb.stanford.edu/).

Among the 560 samples, 12 patients contributed 2 samples and 1 patient 3 samples, meaning that 546 patients [ $52 \%$ men; median age 33 years, interquartile range (IQR) 19-46] were enrolled in the study. Of 28 plasma pools tested, 4 showed detectable viremia (HIV RNA). In three of these pools, one sample showed viremia. The samples positive for HIV RNA were derived from 2 men and 1 woman (aged 46, 23, and 30 years, respectively.) No clinical information was available for the men, whereas the woman had reported fever, myalgia, retro-orbital pain, and headache. Although clinical symptoms were documented for only one case, samples included in the pool were all derived from patients whose blood was collected for serological confirmation of dengue on the basis of the following sentinel clinical manifestations: fever (typically with a duration of 2-7 days) with $\geq 2$ of the following symptoms: nausea, vomiting, rash, myalgia, headache, retro-orbital pain, leukopenia, petechiae, or a positive tourniquet test.

All three samples had high viral loads, and one sample showed the K103N mutation, which causes high-level resistance to first generation non-nucleoside reverse-transcriptase inhibitors. No nucleotide ambiguities were observed in three sequences, and all were subtype B. Serology was negative in two of the three RNA-positive samples. One was indeterminate, showing only reactivity to glycoprotein 41 (gp41) in an immunoblot (Table 1).

Two samples in the fourth positive pool failed automated extraction and were therefore unavailable for further testing. All other samples in this and other positive pools tested as HIV RNA not detected.

Assuming 1 positive sample for each reactive pool (4/546), an HIV prevalence of $0.73 \%$ [ $95 \%$ confidence interval (CI) $0.23-1.76$ ] can be estimated for this sample; this would change to $0.88 \%$ if samples from children $<14$ years old were not computed. This prevalence is almost twice that expected for the general population ${ }^{9}$; however, the CIs do overlap. High viremia, indeterminate or negative serology, lack of ambiguous nucleotides, and no previous registry in electronic systems are compatible with unrecognized and likely acute HIV infections.

In this small study, we could validate the concept that some HIV-infected individuals, possibly during the acute phase of their disease, could be identified during dengue evaluation. Even though we used samples leftover from previous serological tests, the material was suitable for further HIV testing, a procedure that might be optimized. In this study, we only evaluated seronegative samples; however, some positive dengue samples might not represent current illness, particularly in endemic areas, as IgM may be present for up to three months or longer ${ }^{10}$. Moreover, variable specificity was observed. In an evaluation of 4 rapid IgM-based kits, sensitivities and specificities ranged from $21-99 \%$ and $77-98 \%$, respectively, when compared with the gold standard laboratory-based ELISA. Moreover, falsepositive results were present in patients with other tropical diseases or previous dengue infections ${ }^{10}$. Therefore, actual coinfections cannot be ruled out, and it might be useful to evaluate all samples in certain settings.

Along with the use of pooled plasma, which might be costeffective in vulnerable populations, additional strategies to identify cases of acute HIV disease among dengue cases might prove useful. This was also shown in a study in Singapore, where, among patients $>15$ years old suspected of dengue by emergency physicians, 8/294 (2.4\%) acute phase HIV patients were identified; of these, 7 satisfied World Health Organization (WHO) case classification criteria for probable dengue ${ }^{11}$. Strategies to identify acute HIV infection have been proposed ${ }^{12}$ and should be used concurrently to improve the incorporation of these patients into care.

In countries like Brazil that offer free laboratory tests for both dengue and HIV, it would be easier to implement routine pooled strategies at laboratories that perform large-scale testing. Pooled tests with opt-out recall authorization at collection might be suitable for such initiatives. Test results would then be returned during previously scheduled follow-up visits to review dengue results, adapting on capability already in place. The current cost of testing for viral load is higher than that of serology at a similar prevalence scenario. However, the main target for this initiative, patients with high viral loads and acute viral symptoms, likely include cases in which antibodies might not be detectable yet; they might therefore be missed by current rapid testing campaigns. Moreover, targeting cases with high viremia allows sub-pooling strategies, reducing the overall cost per diagnosed case albeit increasing pooling complexity, a limitation that might be overcome with automatization.

Besides the potential for monitoring local epidemics, these cases represent missing opportunities for a more comprehensive

TABLE 1

Demographic and laboratory characteristics of identified HIV infections.

\begin{tabular}{lcccccccc}
\hline Pool number & Sex & Age (yrs) & $\begin{array}{c}\text { Drug resistance } \\
\text { mutation }\end{array}$ & Viral load & HIV subtype & $\begin{array}{c}\text { Nucleotide } \\
\text { ambiguity }\end{array}$ & Immunoblot \\
\hline 10 & Male & 22 & K103N & 6.07 & B & None & Negative \\
11 & Male & 46 & None & 6.12 & B & None & Indeterminate & Gp41 \\
17 & Female & 29 & None & 5.85 & B & None & Negative \\
\hline
\end{tabular}


healthcare approach that could benefit patients and decrease transmission of HIV at this heightened phase of infection.

\section{Conflicts of interest}

The authors declare that there are no conflicts of interest.

\section{Financial support}

This study was partially funded by a FAPESP grant (award number: 2013/19441-7).

\section{REFERENCES}

1. Cohen MS, Shaw GM, McMichael AJ, Haynes BF. Acute HIV-1 infection. N Engl J Med. 2011;364(20):1943-54.

2. Tsai MS, Tsai YT, Pan CT, Fang KH. Acute human immunodeficiency virus infection presenting as mononucleosis-like illness, acute hepatitis, and aseptic meningoencephalitis. J Med Sci. 2012;28(11):628-9.

3. Buchanan JG, Goldwater PN, Somerfield SD, Tobias MI. Mononucleosis-like-syndrome associated with acute AIDS retrovirus infection. N Z Med J. 1986;99(803):405-7.

4. The INSIGHT START Study Group, Lundgren JD, Babiker AG, Gordin F, Emery S, Grund B, Sharma S, et al. Initiation of antiretroviral therapy in early asymptomatic HIV infection. N Engl J Med. 2015;373(9):795-807.
5. Joint United Nations Program on HIV/AIDS (UNAIDS). 90-9090 An ambitious treatment target to help end the AIDS epidemic. Geneve: 2014. 40p. Acessed 13 July 2016. Available in: http://www. unaids.org/en/resources/documents/2014/90-90-90.

6. Pilcher CD, McPherson JT, Leone PA, Smurzynski M, OwenO'Dowd J, Peace-Brewer AL, et al. Real-time, universal screening for acute HIV infection in a routine HIV counseling and testing population. JAMA. 2002;288(2):216-21.

7. Sullivan TJ, Patel P, Hutchinson A, Ethridge SF, Parker MM. Evaluation of pooling strategies for acute HIV-1 infection screening using nucleic acid amplification testing. J Clin Microbiol. 2011;49(10):3667-8.

8. Pilcher CD, Louie B, Facente S, Keating S, Hackett Jr J, Vallari A, et al. Performance of rapid point-of-care and laboratory tests for acute and established HIV infection in San Francisco. PLoS One. 2013;8(12):e80629.

9. Ministério da Saúde. Secretaria de Vigilância em Saúde. Departamento de DST AIDS e Hepatites Virais. Boletim Epidemiológico HIV/AIDS. Brasília: MS; 2015. p. 1-100.

10. Peeling RW, Artsob H, Pelegrino JL, Buchy P, Cardosa MJ, Devi S, et al. Evaluation of diagnostic tests: dengue. Nat Rev Microbiol. 2010; 8 (suppl 12):S30-S37.

11. Verrall A, Tan JH, Archuleta S. Acute retroviral syndrome mimics dengue in Singapore. Ann Acad Med Singapore. 2012;41(12):617-9.

12. Prins HA, Mugo P, Wahome E, Mwashigadi G, Thiong'o A, Smith A, et al. Diagnosing acute and prevalent HIV-1 infection in young African adults seeking care for fever: a systematic review and audit of current practice. Int Health. 2014;6(2):82-92. 